\section{More than meets the eye}

lan Stewart

Theories of Everything: The Quest for Ultimate Explanation. By John D. Barrow. Oxford University Press: 1991. Pp.223. £14.95, \$22.95.

Most scientists work in tiny corners of their subject, content to confine their activities to a few particular facets of the natural world. Others tackle the big problems that they believe lie behind these more mundane activities: the nature of the universe, its ultimate components, its origins. They search for a self-proclaimed 'theory of everything'; and they appear to be increasingly confident that they are about to find it.

Are they right? If they are, how much use will a theory of everything actually be? In his opening chapter, John Barrow remarks that ultimate questions had been falling out of fashion among scientists until recently. Suddenly thinking more appropriate to theology is showing up in the new theories of science, to the extent that theologians are having to come to terms with superstrings. "The theologians think they know the questions but cannot understand the answers. The physicists think they know the answers but don't know the questions. An optimist might thus regard a dialogue as a recipe for enlightenment, whilst the pessimist might predict the likely outcome to be a state in which we find ourselves knowing neither the questions nor the answers."

Any study of why science has returned to speculation on the grand scale, of which its speculations are, and of how they may affect our lives, must also take in many other matters of a philosophical nature. What is scientific explanation? In what sense does it capture the true behaviour of the natural world? Why is mathematics such a powerful weapon in scientific understanding? Theories of Everything is a beautifully written and thoughtful attempt to make sense of this difficult area of enquiry. It is worth buying for its apt and amusing quotations alone, such as Poul Anderson's "I have yet to see any problem, however complicated, which when you looked at it in the right way, did not become still more complicated."

What is a law of nature? The very word is a curious choice, for one cannot break a natural law. Laws are created by mankind to control human behaviour in an imperfect world. There is something rather anthropomorphic in the idea that nature, too, has laws and obeys them. But, without doubt, there is a strong belief, not just that the universe has patterns, but that it has a single unified pattern. We are dissatisfied with distinct theories for distinct realms of nature, however successful each may be: there is something fundamentally disturbing in the philosophical and mathematical inconsistency of quantum mechanics and general relativity. Inconsistency is an unhappy reminder that our 'natural' laws are man- made: what better response than to unite everything in a single law?

The realm of quantum mechanics is that of the electron, that of general relativity is the galactic supercluster. At the tiniest scales, we find particle physics; at the largest, cosmology. But in modern cosmology the worm eats its own tail: the behaviour of fundamental particles is crucial for the first instants of the big bang. In this style of physics, it is impossible not to seek to reconcile the two theories, for, as logicians have always known, speculation within an inconsistent framework can produce any conclusion whatsoever.

Even within a framework of agreed laws, different initial conditions lead to different dynamical evolution. The initial conditions for our universe are probably unknowable, and this is somewhat awkward. Many cosmologists hope that the current state of the universe is largely independent of its initial conditions. Others seek to prescribe the initial conditions as part of the laws; or, bearing in mind that time itself may 'start' along with the embryonic universe, argue that the idea of an initial condition is simply misleading. Relativity complicates the question: causally disconnected regions of spacetime may have independent 'initial' conditions.

The role of initial conditions is very much bound up with the nature of time itself, something that a true 'theory of everything' must address. The same goes for the nature of matter. For instance, why are there so many identical elementary particles in the universe? All electrons are equal. Nothing on a macroscopic scale works this way; no two rocks, planets, clouds or bushes are exactly alike. But down on the tiniest scales, the universe is not as complicated as it might be. The symmetry principles of particle physics must surely be related to this puzzle; but are they a solution, or a consequence?

There is another crucial question that a 'theory of everything' must answer. Why do the various constants of nature (such as the speed of light or the mass of the electron) assume the particular values that we measure? For that matter, why are they constant? Indeed, are they constant? Here the anthropomorphic principle raises its head. Perhaps many of the 'constants' are arbitrary. But for human intelligence in its present form to be possible, they must take values very close to the observed ones. When the sole survivor of a plane crash asks 'why me?' the fallacy is obvious. But if there can be only one universe, the anthropic principle still tends to beg the question. The problem vanishes if we accept that (thanks to wormholes and inflation) there may be other universes. In one of these a quasi-intelligent pseudoplasmoid may be wondering why the speed of light, at 255 metres per hour, is exactly what is needed to make quasi-intelligent pseudoplasmoids possible. Incidentally, the strong anthropic principle, which effectively holds us to be the purpose of the enterprise, looks less compelling when observed through the communicatory field-modulators of a quasiintelligent pseudoplasmoid.

Of such questions (though, I hasten to add, not that particular example) is Theories of Everything built. Its scope is, appropriately, vast; it draws from science, culture, myth and religion; and it touches upon many important concerns, ranging from chaos and symmetry-breaking to information-compression and the dilute wormhole approximation. Its intention is to talk about theories, rather than to explain them in detail, and this occasionally causes difficulties for the casual reader. Some assertions are questionable: for example that an "important lesson we have learnt from the mathematicians' approach to axiomatic systems is that one can quantify the amount of information that is contained in a collection of axioms. None of the possible deductions . . . can possess more information than was contained in the axioms." The spirit of the statement is correct, although I shall argue below that it tends to be misinterpreted, but its qualification through information theory is a red herring.

The author's main conclusion is not about the likely truth of the current attempts at 'theories of everything'. It is that, even if successful, such theories will not, in practice, render everything else obsolete. Yes, another anti-reductionist book. I tend to agree, though perhaps for different reasons. In practice, deductions from axioms - and laws - do 'contain' more information than the axioms themselves. More accurately, they make that information accessible. 'Knowing' laws is different from 'knowing' their consequences, even though the latter may well be implicit in the former. The quantification that is traditional in information theory misses the point here: there is a human cost in making the consequences explicit, and until this is done, the 'information' in the original laws is useless to us. This is why society pays mathematicians to make deductions that add zero information: it wants to know what the answers are, not just that they are implicit in the problem.

Similarly, physicists believe that the existence of crystalline states of matter is a consequence of quantum mechanics; but nobody can yet prove this mathematically. Until the question is decided, we do not know whether the 'information' in crystallography adds anything to that in quantum mechanics. But, whatever the answer, crystallographers will continue to use crystallography to study crystals. As Barrow so rightly says: "There is more to Everything than meets the eye." $\square$

Ian Stewart is in the Mathematics Institute, University of Warwick, Coventry CV4 7AL, UK. 